\title{
Self-infusion with immunoglobulin at home
}

\author{
H Chapel, V Brennan
}

Primary hypogammaglobulinaemia is rare but is easily diagnosed and treated. It is still frequently missed due to the wide variety of presenting symptoms which may involve many different medical and surgical specialities. Its prevalence in the United Kingdom is unknown but is probably between 1 and 2 per 50000 population, based on Swedish figures. ${ }^{1}$

Treatment with replacement immunoglobulin is essential for patients who are unable to make antibodies due to hypogammaglobulinaemia. It is also successful in preventing infection in those patients with less obvious but demonstrable deficiencies, such as IgG subclass deficiencies or specific antibody failure. Intravenous immunoglobulin has largely replaced intramuscular preparations, though this route is still appropriate for mildly affected patients. Greater compliance, however, painless infusions, and the ability to give large doses of immunoglobulin make intravenous immunoglobulin the treatment of choice in most hypogammaglobulinaemic patients. That such infusions are effective in preventing infections, even in patients with severe chest disease, is in no doubt. ${ }^{23}$

The present (third) generation of intravenous preparations provide unmodified (native) IgG with a wide range of antibodies to bacteria and viruses as well as good opsonising ability. Furthermore, these preparations have a low incidence of adverse reactions and it is this that has enabled patients to have intravenous $\mathrm{Ig}$ infusions safely in their own homes. The motivation for self-infusion comes from the patients themselves because they are able to avoid time off work for hospital visits as well as being more involved in their own disease and its treatment. The financial savings to the hospital, despite requirements of a nursing sister to run a home treatment programme, also provides additional motivation to immunologists and hospital administrators to set up such programmes. ${ }^{4}$

The first self-infusion programmes were started in the United States ${ }^{56}$ and a pilot study was done in the United Kingdom in 1986-87 to test the feasibility and safety of self-infusion at home. ${ }^{4} \mathrm{~A}$ United Kingdom register of patients who are self-infusing at home has been kept in Oxford and over 100 patients from six centres have been entered into this register so far. Several other centres have started home treatment programmes for hypogammaglobulinaemic patients; training courses are regularly held in Oxford, London, Birmingham, Manchester and Bristol. Patients in Newcastle and Edinburgh also self-infuse, and it is hoped that other centres will develop such programmes in due course.
Guidelines for centres setting up home immunoglobulin treatment programmes have been drawn up and have recently been approved not only by the Association of Clinical Pathologists but also by the Royal College of Physicians and the Royal College of Pathologists. The Department of Health have considered the guidelines, and both supported the concept of home treatment and recommended that such programmes are set up on a regional basis.

Home treatment involves a considerable commitment by both medical, nursing, and secretarial staff. Training in self-infusion techniques alone is not enough. Close contact with the centre for immediate problems and arrangements of follow up is crucial. Safeguards against non-compliance must be built into the programme as should support for patients with poor veins or loss of confidence associated with domestic crises. General practitioners are interested in the home programme and their consent must be obtained. In our experience general practitioners have been most helpful on the rare occasions when immediate advice on site has been needed.

No serious adverse reactions have occurred in any of the patients while self-infusing. This is a reflection of the careful selection of the patients for the programme. It is important that they have received intravenous immunoglobulin for at least six months before acceptance on the programme and that they have sustained no adverse reactions during this time. Additional criteria include the availability of a partner (spouse, parent, or friend) who is willing to be trained and to help on all occasions, good veins, reasonable manual dexterity and understanding of their disease and treatment. Non-compliance is an obvious contraindication. Though age is no bar to selfinfusion, older patients (those over 50 years) do take longer to train and to become fully confident ${ }^{4}$; once trained they have remained confident and infusions have been uneventful.

The age range of the patients in the Oxford programme is considerable. Although our experience of children is still limited, the youngest patient trained so far is 7 years of age. Experience with haemophiliacs, however, suggests there is no reason why younger children should not allow their mother or father to perform venepuncture. We hope that paediatricians managing children with hypogammaglobulinaemia, as well as paediatric immunologists, will encourage other families to join home treatment programmes.

The training course in Oxford takes two or three days; there is a great deal to cover, but attendance at a formal course is a legal requirement to protect both patient and doc- 
tor. Not only are techniques of aseptic reconstitution of the immunoglobulin, setting up the pooling bag, priming the giving set and venepuncture taught and practised, but patients must be taught how to recognise possible adverse reactions and how to treat them appropriately. Patients take their own blood samples and send them regularly for IgG measurements and liver function tests. They must be able to complete infusion logs and daily diaries to enable us to monitor acute infections. It is essential that a partner is also trained and available to provide support in case an unexpected adverse reaction should occur.

Once trained, patients and their partners perform the next few infusions under medical supervision. Once they and their immunologists are confident that they are safe to infuse at home, home infusions can begin with the general practitioner's approval. The provision of the immunoglobulin preparations and the infusion consumables at home has not proved a problem because immunoglobulin treatment has followed other forms of home treatment. These include factor VIII infusions, parenteral nutrition, and chronic ambulatory peritoneal dialysis; more recently intravenous antibiotic treatment ${ }^{7}$ has been done at home too. The introduction of GP budgets has caused some anxiety about prescribing replacement treatment, but the Department of Health has made it clear that allowance is made for these unusual but expensive dis- eases. No one would deny immunoglobulin replacement treatment to these patients and self-infusion at home is the most cost effective way of providing it.

The benefits to patients receiving intravenous treatment at home are obvious. Increased convenience and the minimal disruption to their family and working lives improves morale, both for themselves and their families. Increased involvement in their treatment also raises self-esteem. Self-administration of intravenous immunoglobulin at home has proved safe and it is hoped that it will be the method of choice for replacement treatment for most patients with hypogammaglobulinaemia in the United Kingdom, currently estimated to be between 1-2000 patients.

1 Bjorkander J, Bake B, Hanson L. Primary hypogammaglobulinaemia impaired lung function and body growth with delayed diagnosis and inadequate treatment. Eur $J$ Resp Dis 1984;65:529-36.

2 Roifman C, Levison H, Gelfand E. High-dose versus lowdose intravenous immunoglobulin in hypogammaglobulinaemia and chronic lung disease. Lancet 1987; 1075-7.

3 Eibl M, Wedgewood R. Intravenous immunoglobulin: A review. Immol Rev 1989;1:[S] 1-42.

4 Chapel HM, Brennan V, Delson E. Immunoglobulin replacement therapy by self infusion at home. Clin Exp Immunol 1988;73:275-7.

5 Ochs HD, Fischer SH, Lee ML, Delson E, Kingdom HS Wedgewood RJ. Intravenous immunoglobulin home treatment for patients with primary immunodeficiency disease. Lancet 1986;i:610.

6 Ashida ER, Saxton A. Home intravenous immunoglobulin therapy by self administration. J Clin Immunol 1986;6: 306-9.

7 Winter RJ, George RJ, Deacock SJ, Shee CD, Geddes DM. Self-administration home intravenous antibiotic therapy in bronchiectasis and adult cystic fibrosis. Lancet 1984; ii:1138-9. 